\title{
The Emerging Importance of Skeletal Muscle Function in Assessing \\ Candidates for Transplantation
}

James R Walsh, BPhty, PhD ${ }^{1,2,3}$, Daniel C Chambers MRCP, FRACP, MD ${ }^{2,4}$, and Peter MA Hopkins MBBS, FRACP ${ }^{2,4}$

${ }^{1}$ Physiotherapy Department, The Prince Charles Hospital, Brisbane, Queensland, Australia, 4032, ${ }^{2}$ Queensland Lung Transplant Service, The Prince Charles Hospital, Brisbane, Queensland, Australia, 4032, ${ }^{3}$ School of Rehabilitation Sciences, Griffith University, Gold Coast, Queensland, Australia, 4215 and ${ }^{4}$ School of Medicine, The University of Queensland, Brisbane, Queensland, Australia, 4072

\section{Correspondence:}

Dr James Walsh

Physiotherapy Department

The Prince Charles Hospital

Rode Road

Chermside, 4032

Queensland

Australia

Email address: james.walsh@ health.qld.gov.au 
Authorship page:

James R Walsh contributed to the drafting of the manuscript and final approval. (Email address: james.walsh@health.qld.gov.au).

Daniel C Chambers contributed to the drafting of the manuscript and final approval (Email address: daniel.chambers@ health.qld.gov.au).

Peter MA Hopkins contributed to the drafting of the manuscript and final approval. (Email address: peter.hopkins@ @ealth.qld.gov.au)

Authors declare no conflict of interest 
Skeletal muscle function is an increasingly important component of assessing candidates' suitability for transplantation. Functional status and physiological age have been proposed as significant considerations in the selection of candidates for lung ${ }^{(1)}$ and liver transplantation. ${ }^{(2)}$ Severely limited functional status with poor rehabilitation potential is now considered an absolute contraindication in the selection of lung transplant candidates. ${ }^{(1)}$ Furthermore, the place of lung transplantation in candidates with age greater than 65 years in association with low physiologic reserve and or other relative contraindications should be very carefully assessed as outcomes are inferior. ${ }^{(1)}$ Physiological rather than chronological age, along with assessment of functional status, have been recommended as considerations when assessing an older candidate's suitability for liver transplantation. ${ }^{(2)}$ Functional status is a multidimensional measure that should include assessment of both functional capacity and functional performance ${ }^{(3)}$ with skeletal muscle function impacting upon both. However, defining what are the optimal measures in assessing a transplant candidates' functional status and a suitable threshold of functional status for active transplant listing is complex.

The recent international heart transplantation listing criteria suggest that the assessment of frailty may be considered when assessing candidacy. ${ }^{(4)}$ Frailty has been explained as a biologic syndrome of decreased reserve and resistance to stressors, resulting from cumulative declines across multiple physiologic systems and causing susceptibility to adverse outcomes. ${ }^{(5)}$ Frail skeletal muscle, or sarcopenia, defined as a progressive loss of skeletal muscle mass, strength and power, is regarded as a key component of frailty ${ }^{(6)}$ However, it is not yet clear which skeletal muscles should be assessed and the minimal threshold of muscle function required prior to a candidate being considered suitable for transplantation. 
Rozenberg and colleagues should be congratulated for prospectively assessing multiple measures of muscle function in 50 lung transplant candidates and evaluating the association of these measures with exercise capacity, activities of daily living and healthrelated quality of life. ${ }^{(7)}$ Skeletal muscle deficits were defined as low muscle mass using bioelectrical impedance; reduced strength assessed by quadriceps peak torque, hand grip force, respiratory muscle strength; and or a lower physical performance using the Short Physical Performance Battery. ${ }^{(7)}$ Low quadriceps muscle strength and handgrip force were defined as $>1$ standard deviation below predicted values with a score of $\leq 9$ in the Short Physical Performance Battery being used to define low physical performance. ${ }^{(7)}$ The study participants had completed pulmonary rehabilitation prior to assessment. Rozenberg and colleagues' primary aims were to characterize any skeletal muscle deficits and determine if these deficits were associated with exercise capacity using the 6 minute walk distance (6MWD), activities of daily living and health-related quality of life. ${ }^{(7)}$ The study had a secondary aim of assessing associations between muscle deficits and pre and early posttransplant clinical outcomes including 6MWD at 3 months' posttransplantation. ${ }^{(7)}$

Rozenberg and colleagues found that skeletal muscle deficits in their prelung transplant cohort were characterized predominantly by reduced quadriceps muscle strength and, to a lesser extent, a lower score in the Short Physical Performance Battery. ${ }^{(7)}$ Quadriceps muscle strength and the Short Physical Performance Battery score were both associated with pretransplant 6MWD, activities of daily living and health-related quality of life. ${ }^{(7)}$ The authors also found that lung transplant candidates with $\geq 2$ muscle deficits had significantly lower 6MWD, activities of daily living and health-related quality of life than candidates without any skeletal muscle deficits. ${ }^{(7)}$ Both reduced quadriceps muscle strength and an increase in the total number of skeletal muscle deficits were associated with longer 
posttransplant hospital length of stay but not intensive care duration in univariable analysis. ${ }^{(7)}$ These findings are of interest, as they not only highlight the importance of assessing skeletal muscle function in lung transplant candidates but also suggest that assessment of quadriceps muscle strength and the administration of the Short Physical Performance Battery may be of most value.

Recovery of quadriceps muscle strength has been strongly associated with recovery in exercise capacity after lung transplantation, ${ }^{(8,9)}$ and in heart transplant recipients' improvements in exercise capacity after exercise training have been found to be related to improvements in muscle recovery. ${ }^{(10)}$ However, Rozenberg and colleagues found that candidates' quadriceps muscle strength and the total number of skeletal muscle deficits were not associated with 6MWD at 3 months' posttransplantation. ${ }^{(7)}$ One explanation for this finding is the cross-sectional nature of the study design where candidates' skeletal muscle function was assessed at 4 weeks after active transplant listing rather than at the time of the posttransplant 6MWD. It would be a reasonable expectation that there are changes in skeletal muscle function postassessment from either ongoing pretransplant rehabilitation, the waitlist duration or the early posttransplant course ${ }^{(8,9)}$ which may have impacted on these results. This limitation in the cross-sectional study design is potentially important as we have previously demonstrated that recovery in recipients' quadriceps strength was an independent predictor of improvement in 6MWD during the first 26 weeks after lung transplantation. ${ }^{(9)}$ Future research should assess the impact of longitudinal changes in skeletal muscle function in transplant candidates and their influence on posttransplant outcomes. 
A limitation of the study by Rozenberg and colleagues was that skeletal muscle function was only assessed in 50 lung transplant candidates with the study assessing a relatively large number of people with interstitial lung disease $(64 \%){ }^{(7)}$ The small sample size somewhat limits the conclusions which can be drawn from their work and precluded multivariable analysis. The large proportion of patients with interstitial lung disease means that the study's findings may not be applicable to other lung transplant programs with a different case mix. Further research will be required to assess the generalisability of their findings.

In conclusion, Rozenberg and colleagues' findings point to assessment of quadriceps muscle strength and the administration of the Short Physical Performance Battery as being particularly important when assessing muscle function in the lung transplant candidate. Further research is required to determine if targeted interventions to candidates' skeletal muscle deficits can improve transplant outcomes. 


\section{References}

1. Weill D, Benden C, Corris PA, et al. A consensus document for the selection of lung transplant candidates: 2014--an update from the Pulmonary Transplantation Council of the International Society for Heart and Lung Transplantation. J Heart Lung Transplant. 2015; 34(1):1-15.

2. Martin P, DiMartini A, Feng S, Brown R Jr, Fallon M. Evaluation for liver transplantation in adults: 2013 practice guideline by the American Association for the Study of Liver Diseases and the American Society of Transplantation. Hepatology. 2014; 59(3):1144-65.

3. Spruit MA, Singh SJ, Garvey C, et al. An official American Thoracic Society/European Respiratory Society statement: key concepts and advances in pulmonary rehabilitation. Am J Respir Crti Care Med. 2013; 188(8):e13-64.

4. Mehra MR, Canter CE, Hannan MM, et al. The 2016 International Society for Heart Lung Transplantation listing criteria for heart transplantation: A 10-year update. $J$ Heart Lung Transplant. 2016; 35(1):1-23.

5. Fried LP, Tangen CM, Walston $J$, et al. Frailty in older adults: evidence for a phenotype. J Gerontol A Biol Sci Med Sci. 2001; 56(3):M146-56.

6. Clegg A, Young J, Iliffe S, Rikkert MO, Rockwood K. Frailty in elderly people. Lancet. 2013; 381(9868):752-62. 
7. Rozenberg D, Singer LG, Herridge M, et al. Evaluation of skeletal muscle function in lung transplant candidates. [published online April 3 2017]. Transplantation. doi: 10.1097/TP.0000000000001754.

8. Maury G, Langer D, Verleden G, et al. Skeletal muscle force and functional exercise tolerance before and after lung transplantation: a cohort study. Am J Transplant. 2008; $8(6): 1275-81$.

9. Walsh JR, Chambers DC, Davis RJ, et al. Impaired exercise capacity after lung transplantation is related to delayed recovery of muscle strength. Clin Transplant. 2013; 27(4):E504-11.

10. Haykowsky M, Taylor D, Kim D, Tymchak W. Exercise training improves aerobic capacity and skeletal muscle function in heart transplant recipients. Am J Transplant. 2009; 9(4):734-9. 